EPJ manuscript No.

(will be inserted by the editor)

\title{
Transport of a quantum degenerate heteronuclear Bose-Fermi mixture in a harmonic trap
}

\author{
C. Klempt, T. Henninger, O. Topic, J. Will, St. Falke, W. Ertmer, and J. Arlt \\ Institut für Quantenoptik, Leibniz Universität Hannover, Welfengarten 1, D-30167 Hannover, Germany
}

October 29, 2018

\begin{abstract}
We report on the simultaneous transport of mixed quantum degenerate gases of bosonic ${ }^{87} \mathrm{Rb}$ and fermionic ${ }^{40} \mathrm{~K}$ in a harmonic potential. The samples are transported over a distance of $6 \mathrm{~mm}$ to the geometric center of a Ioffe-Pritchard type magnetic trap. This transport mechanism was implemented by modification of the QUIC trap and is free of losses and heating. It significantly extends the capabilities of this trap design. We demonstrate a launching mechanism for quantum degenerate samples and show that highly homogeneous magnetic fields can be created in the center of the QUIC trap. The transport mechanism may also be cascaded to cover even larger distances for interferometric experiments with quantum degenerate samples.
\end{abstract}

PACS. 52.55.Jd Magnetic mirrors, gas dynamic traps - 03.75.Be Atom and neutron optics - 03.75.Pp Atom lasers - 03.75.Ss Degenerate Fermi gases

\section{Introduction}

Within the past decade, the field of ultracold atomic gases has significantly extended the scope of atomic and molecular physics [123]. The experimental manipulation of quantum degenerate gases has led to the development of a tool' box for quantum atom optics [4] including guides [5], beam splitters and combiners [6] as well as switches [7]. These tools are intended for the development of a new generation of guided interferometric sensors 8 8|9|10|11|12]13|14. One of the main requirements for such experiments is the coherent spatial transport of quantum degenerate gases. At present, interferometric applications involving ultracold fermionic atoms are also evaluated and, in some cases, regarded as superior compared to their bosonic counterpart [15[16]17. However, adiabatic transport of quantum degenerate fermionic samples had not been accomplished up to now.

Three main types of mechanical transport mechanisms for Bose-Einstein condensates over macroscopic distances have been reported. The first transport was achieved by carefully moving the focus of a red-detuned dipole trap over a distance $44 \mathrm{~cm} \mathrm{18}$. Although the atomic cloud was heated due to vibrations of the moving optical components, the finite depth of the dipole trap provided continuous evaporative cooling. Thus quantum degeneracy was maintained during the transfer time of 7.5 seconds. An alternative method transports the condensate within a one-dimensional optical lattice [19]. In order to obtain long transport distances, the lattice was produced by two counter-propagating Bessel beams. By adjusting the relative phase, it was possible to shift the lattice sites and transport a condensate over up to $10 \mathrm{~cm}$. The third method involves lithographic conducting structures, so-called atom chips. After creating a Bose-Einstein condensate with such a wire structure, it is possible to shift the condensate by applying modulated currents to an additional periodical wire pattern 20]2122. Transfer distances of up to $1.6 \mathrm{~cm}$ were demonstrated.

However, all of these methods suffer from strong heating or loss mechanisms. A moving dipole trap involves the translation of optics and induces heating due to vibration. Optical lattices and chip traps typically produce strongly confining traps with high trapping frequencies and high atomic densities. Although high densities support fast evaporation to quantum degeneracy, they are impractical for the transport because of heating and atom loss due to enhanced three-body collision rates.

In this paper, we report on the transport of quantum degenerate samples of bosonic ${ }^{87} \mathrm{Rb}$ and fermionic ${ }^{40} \mathrm{~K}$ in a harmonic potential over a distance of $6 \mathrm{~mm}$. This also constitutes the first transport experiment with a quantum degenerate Fermi gas. The transport is realized by adiabatically transforming a Ioffe-Pritchard type magnetic trap produced by macroscopic coils. The atom numbers are considerably larger than in experiments with atom chips [22] and the trapping strength is adjustable without changing the trap depth.

The initial production of a quantum degenerate BoseFermi mixture is performed in a so-called QUIC trap, consisting of a pair of anti-Helmholtz coils and a third coil in perpendicular orientation [23]. The adiabatic transport is realized by adding a second anti-Helmholtz pair. A numerical simulation is used to calculate optimized currents 
Klempt et al.: Transport of a quantum degenerate heteronuclear Bose-Fermi mixture in a harmonic trap

for all coils in order to create a slowly changing trap and a smooth transport to the final position while maintaining a magnetic field for proper spin orientation of the sample. By these means, it is also possible to accelerate and launch degenerate ensembles with high precision and reproducibility. This capability is of great interest for its application in fountain clocks 24 ] and inertial sensors [25].

The transport mechanism presented in this paper is particularly useful for applications where low heating rates and large atom numbers are required. In particular, it may be used to load chip traps or optical interferometers [26] with large atomic samples. It may also be used to transport atoms to probe specific position dependent quantities [27. By cascading the coil configuration used in this experiment, it will be possible to cover much larger transport distances.

In our case, the transport is used to load the atomic cloud into a dipole trap located at the geometric center of the QUIC trap. Transporting the cloud to this position enables us to use the coils of the QUIC trap to generate strong homogeneous magnetic fields with small spatial inhomogeinity. Hence, the applicability of the popular QUIC trap is drastically improved, since it can be used to produce strong magnetic fields (around $1000 \mathrm{G}$ ) at moderate currents. The use of such fields has recently become important for the experimental manipulation of the scattering properties of ultracold ensembles in the vicinity of Feshbach resonances [28/29/30/31.

The paper is organized as follows. We give an overview of our experimental setup in Sec. 2] Details of our implementation and results of the transport of quantum degenerate gases are discussed in Sec. 3. We conclude with an outlook in Sec. 4 .

\section{Experimental Setup}

The apparatus used for the experiments described here consists of two glass cells divided by a differential pumping stage: a MOT cell, where the atomic clouds are collected initially, and a science cell where experiments with ultracold atoms are performed (see Fig. 1). The MOT region, designed for the collection of large clouds of $\mathrm{K}$ and $\mathrm{Rb}$, has been described in detail previously 32 .

Atoms are transferred between these two regions of the experiment, by transporting them in a movable magnetic quadrupole trap. This transport mechanism is described in detail, since it is a key element for further transport experiments with quantum degenerate samples.

\subsection{Dual species MOT design}

The MOT is produced in a large glass cell with inner dimensions $50 \mathrm{~mm} \times 50 \mathrm{~mm} \times 140 \mathrm{~mm}$ at a pressure of $1 \times 10^{-9}$ mbar. The cell allows for trapping beams with a diameter of $3 \mathrm{~cm}$ to capture a large number of atoms. Commercial rubidium dispensers and potassium dispensers constructed according to Ref. 33. are used to

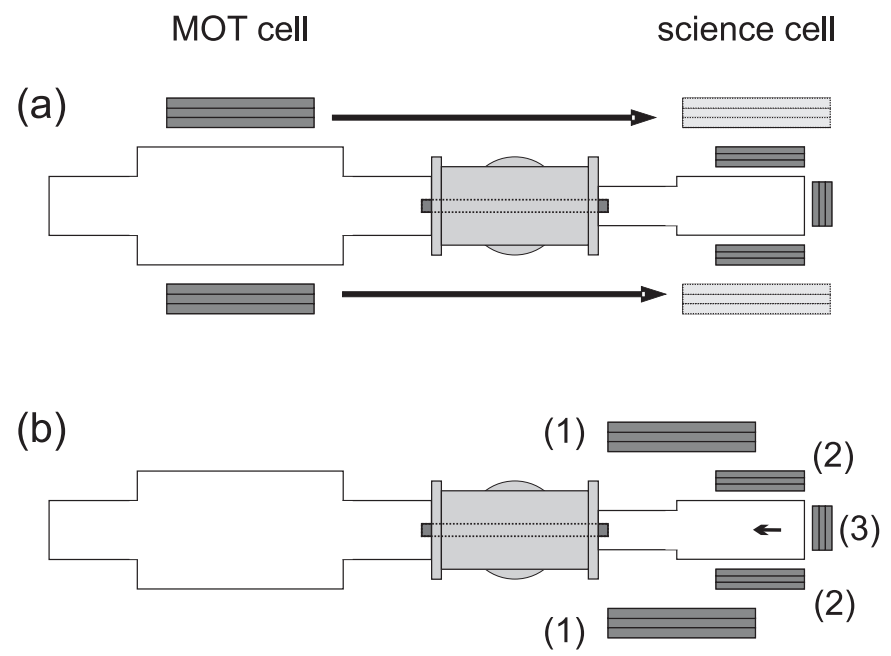

Fig. 1. Outline of the vacuum system and magnetic field coils. The glass cell of the MOT is on the left hand side. The atoms are transported to the science cell on the right hand side by a magnetic field induced by a moving pair of coils as illustrated in (a). A differential pumping stage enables a lower pressure in the science cell. The three coils of the QUIC trap around the science cell are shown on the right hand side. The configuration (b) is used for the transport of the cold mixture to the geometric center of the QUIC trap. The pair of transport coils (1) is displaced from the geometric axis defined by the main coils of the QUIC trap (2), opposite to the QUIC coil (3).

provide vapors of ${ }^{87} \mathrm{Rb}$ and ${ }^{40} \mathrm{~K}$. These dispensers are located at a distance of $35 \mathrm{~cm}$ from the MOT cell and coat the surfaces of the chamber with rubidium and potassium.

Two high power laser systems are necessary to provide the light for magneto-optical trapping of the two atomic species. The cooling and repumping light for the rubidium atoms is provided by two external cavity diode lasers. Both beams are superposed and simultaneously amplified by a tapered amplifier (TA) chip 34. A further external cavity diode laser amplified by a TA provides resonant light at the ${ }^{39} \mathrm{~K}$ D2 transition frequency. This light is divided into two parts and one part is shifted to the ${ }^{40} \mathrm{~K}$ cooling frequency by an acousto-optical modulator (AOM) in double-pass configuration. The second part is tuned resonant to the ${ }^{40} \mathrm{~K}$ repumping by an AOM in quadruple-pass configuration and recombined with the cooling light. After further amplification of the light for potassium with a second TA, it is combined with the light for rubidium with a long pass mirror. A single polarization maintaining fiber collects all four frequencies for the cooling of potassium and rubidium. The use of a single fiber greatly facilitates all further adjustments and the optic setup is not more complicated than for a single species MOT. We operate the MOT with a total power of $360 \mathrm{~mW}$ for $\mathrm{Rb}$ and $160 \mathrm{~mW}$ for $\mathrm{K}$.

The performance of this MOT is further improved by the use of light-induced atom desorption (LIAD) at a wavelength of $395 \mathrm{~nm}$. Atoms that are adsorbed at the walls of a vacuum chamber are desorbed by irradiation 
with weak incoherent light. This allows for a temporary increase of the desired partial pressure. LIAD can thus be used to load a rubidium MOT 35 and to obtain high loading efficiencies 36. Our recent experiments 32 have shown that LIAD is particularly well suited as a switchable atom source, since the pressure decays back to equilibrium after the desorption light is turned off. For the experiments described here about $1 \times 10^{8}{ }^{40} \mathrm{~K}$ and $5 \times 10^{9}$ ${ }^{87} \mathrm{Rb}$ are trapped while the desorption light is on, then they are held in the MOT without desorption light while the pressure drops and finally, only magnetic fields are used to confine them.

\subsection{Magnetic transport}

After the desorption light is switched off, the temperature of the atoms is brought close to the recoil limit in an optical molasses phase. In a second preparation step the atoms are optically pumped to the fully stretched states $\left|f, m_{f}>=\right| 2,2>$ for $\mathrm{Rb}$ and $\mid 9 / 2,9 / 2>$ for $\mathrm{K}$. This allows to capture the atoms in a magnetic quadrupole field induced by two MOT coils. These are mounted on a translation stage. Just before it starts moving, the current of these coils is ramped up from $14 \mathrm{~A}$ to $28 \mathrm{~A}$ in $300 \mathrm{~ms}$, which compresses the cold ensemble.

The coils are moved over a distance of $42 \mathrm{~cm}$ to the science cell at a pressure of $2 \times 10^{-11}$ mbar (see part (a) in Fig. 11). Such transport systems for cold atoms have previously been realized using moving coils 37] or sets of overlapping coils 38, since these systems do not require a second MOT in the science cell and provide far better optical access in this region.

The magnetic confinement for the transport of cold thermal samples is provided by the same coils which produce the small field gradient for the MOT. These coils can produce a quadrupole field with a gradient of up to $138 \mathrm{G} / \mathrm{cm}$. Each coil has 132 turns of $1 \mathrm{~mm} \times 2.5 \mathrm{~mm}$ copper wire. The coils of $13 \mathrm{~mm}$ thickness are separated by $74 \mathrm{~mm}$ and have an inner diameter of $45 \mathrm{~mm}$. Their wires are fixed by epoxy resin to avoid drifts in the performance. No active cooling is needed.

This pair of coils is mounted on a translation stage (Parker, 404 XR series), with a nominal position reproducibility of $5 \mu \mathrm{m}$. However, the motor of the translation stage is switched off if it is at rest to avoid rf noise between 1 and $30 \mathrm{MHz}$, which perturbs the rf evaporation of rubidium. The switching does not effect the position. This experimental technique may be used to transport atoms of two species together [39] or for uniting cold clouds [40].

The translation stage is a reliable, maintenance free tool in our experiments. Although the motion can be controlled in detail, we have chosen a simple operation method. We allow for a maximal speed of $10 \mathrm{~m} / \mathrm{s}$, limit the acceleration to $1 \mathrm{~m} / \mathrm{s}^{2}$, and the jerk to $100 \mathrm{~m} / \mathrm{s}^{3}$. With these settings, the distance of $0.42 \mathrm{~m}$ is covered in less than $1.3 \mathrm{~s}$. Similarly to other experiments 4138, at least one third of the particles reach the science cell. The losses can be accounted to collisions with the background gas and to the transport through the differential pumping tube between the two glass cells. Moreover, we have not observed heating during the transport and can conclude that this type of transport is well suited for the transport of mixtures of different species.

The mechanical transport has been suggested for mixing of cold gases with many components, which may be created in different MOT regions and combined with moving coils 40. This may be especially useful for combinations, which are more difficult to combine in a MOT than $\mathrm{K}$ and Rb. Moreover, a chain of such traps has been suggested as a step towards a continuously created BEC [42].

\subsection{Production of quantum degenerate gases}

The efficient transfer of cold atoms from the MOT region into a harmonic trap in the science cell enables the production of quantum degeneracy for both ${ }^{87} \mathrm{Rb}$ and ${ }^{40} \mathrm{~K}$ by forced rf-evaporation of rubidium. Therefore, the quadrupole field for the transport is converted into a harmonic trapping potential, produced by a magnetic trap in QUIC configuration [see Graph (b) in Fig. 1].

To load the atoms into the QUIC trap, they are first transfered from the transport coils into an even stronger quadrupole formed by the main coils of the QUIC trap. Subsequently, the current through the QUIC coil is ramped up. Thus, the atoms are pulled towards this coil and the trap is deformed such that effective evaporation to quantum degeneracy is possible [23. The ${ }^{87} \mathrm{Rb}$ atoms are cooled by rf-evaporation until a Bose-Einstein condensate with up to $1.5 \times 10^{6}$ atoms at a temperature $T=460 \mathrm{nK}$ with a transition temperature $T_{\mathrm{C}}=580 \mathrm{nK}$ is reached. The ${ }^{40} \mathrm{~K}$ atoms are cooled sympathetically with the $\mathrm{Rb}$ atoms down to the same temperature reaching a quantum degenerate Fermi gas with $1.3 \times 10^{6} \mathrm{~K}$ atoms (with a Fermi temperature of $T_{\mathrm{F}}=1530 \mathrm{nK}$ ).

The QUIC trap consists of a set of coils in anti-Helmholtz configuration with 92 turns with a separation of $30 \mathrm{~mm}$ and a third QUIC coil with 86 turns [see (2) in Graph (b) of Fig. 1, which is offset from the center of the anti-Helmholtz pair by $40 \mathrm{~mm}$ [see (3) in Graph (b) of Fig. 1. This coil configuration produces an offset field of $1.4 \mathrm{G}$ and trapping frequencies for ${ }^{87} \mathrm{Rb}$ of $23 \mathrm{~Hz}$ axially and $240 \mathrm{~Hz}$ radially. In this configuration the same current of 25 A flows through all three coils, yielding an offset field stability of $3 \mathrm{mG}$. All experimental results presented in this paper were acquired by releasing the atomic clouds from the magnetic confining potential and taking resonant absorption images after ballistic expansion.

\section{Transport in a harmonic trap}

Many recent experiments with cold ensembles utilize homogeneous magnetic fields to manipulate the interaction of cold atoms in the vicinity of Feshbach resonances [43]. In our experiments the homogeneous magnetic fields are created by the main coils of the QUIC trap [31, a solution 


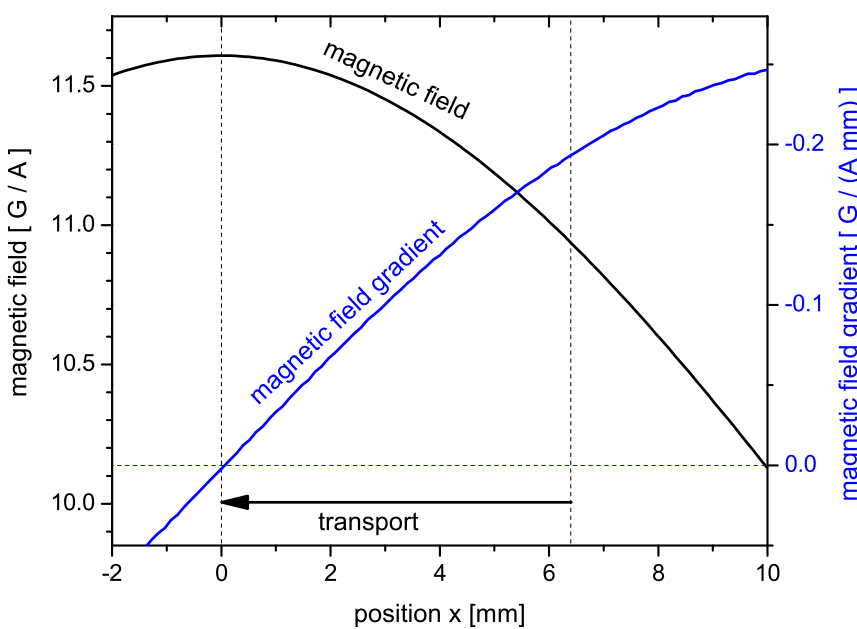

Fig. 2. Calculated magnetic field strength and its gradient generated by the main coils of the QUIC trap operated in Helmholtz configuration as a function of the radial position in the symmetry plane. The transport to the geometric center of the QUIC trap results in a smaller variation of the magnetic field strength over the size of the size of the cloud.

that reduces the experimental complexity in the proximity of the cell. This allows us to profit from the achieved high mechanical and current stability of the QUIC trap.

However, any displacement of the atoms from the symmetry axis leads to a variation of the magnetic field over the width of the cloud. This variation is much smaller in the geometric center of the pair of main coils as shown in Fig. 2. A transport of the cold mixture to this region will therefore lead to a smaller magnetic field spread and thus a better control of this crucial parameter.

We have implemented such a transport of ultracold or quantum degenerate samples over a distance of $\approx 6 \mathrm{~mm}$ in a harmonic trap. This transport is realized by extending the capabilities of a magnetic trap in QUIC configuration 23] with an additional coil pair in anti-Helmholtz configuration. In our case that coil pair is identical to the one for the transport to the science cell. Part (b) of Fig. 1 illustrates the coil configuration around the vacuum system for this transport.

The basic idea is to obtain a quadrupole field induced by the two coil pairs (main and transport), which is shifted away from the one induced by the main coils alone. For any position, the QUIC coil can be used to convert the effective quadrupole field to a harmonic trap.

We have simulated the magnetic fields to obtain optimal parameters for the timing of all currents to realize the transport. The position of the transport coils was chosen such that full optical access to the cell is guaranteed. Precise control of the translation stage enables us to position the atoms exactly in the center of the main coils.

For the transport of a quantum degenerate atomic ensemble in the magnetic trap, one has to consider both the position of the potential minimum and the offset field, which corresponds to the trapping frequencies. In particular, the offset field should never drop to zero during the transformation, otherwise spin flips quickly lead to

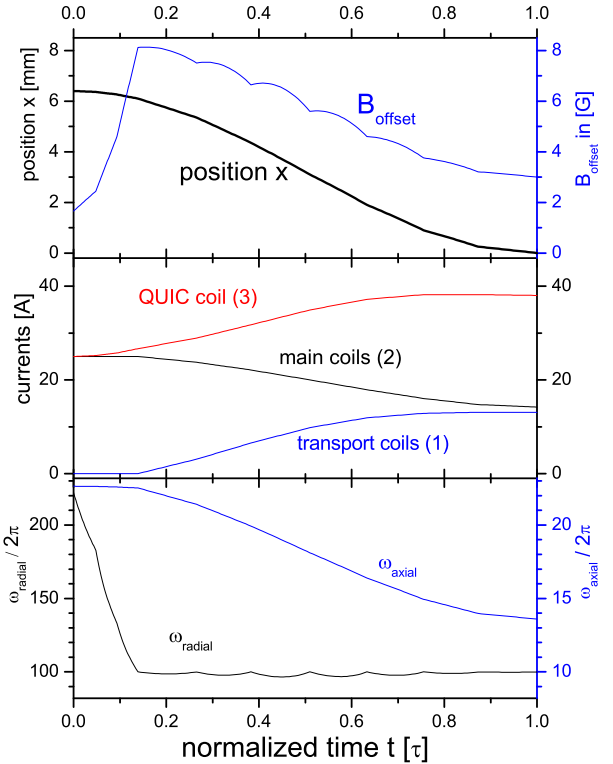

Fig. 3. Simulation of the transport from the QUIC trap to the symmetry axis of the quadrupole coils. The top graph shows the resulting positions and the offset magnetic field. The middle graph illustrates the currents as obtained in an optimization with twelve points. The wobbles for the magnetic field are due to the linear interpolation between these points. The trap frequencies for ${ }^{87} \mathrm{Rb}$ are depicted in the bottom graph. Note that the trap frequencies for ${ }^{40} \mathrm{~K}$ can be interfered directly using the ratio of the masses since the product $m_{f} g_{f}=1$ for the respective transported states is equal.

loss from the trap and destroy a quantum degenerate ensemble. Another goal of our optimization strategy was to quickly reduce the radial trapping frequency in order to minimize density dependent losses and heating.

An optimization algorithm was applied to find suitable currents through the coils taking these issues into account. We aimed for the following functional behavior of the position $x$ with time $t$

$$
x(t)=D \frac{1}{2}\left[\cos \left(\pi \frac{t}{\tau}\right)+1\right]
$$

for a total transfer from position $D$ to the origin in the time $\tau$. Thus, the velocity of the trap changes steadily from and to zero. The result of the simulation is shown in Fig. 3. To realize the transfer, the current of the additional quadrupole coil pair and the current through the QUIC coil are simultaneously increased while the current through the anti-Helmholtz coil pair is decreased. The center of the harmonic potential shifts towards the common center of the two quadrupole coil pairs.

By controlling the currents through the coils, the transfer can in principle be realized adiabatically. Since it is experimentally necessary to implement the time dependence of the currents in linear ramps we have limited our simulation to twelve linear ramps.

Based on this simulation, we have experimentally implemented the transport and took absorption images after each of the applied ramps. The atoms follow the position 


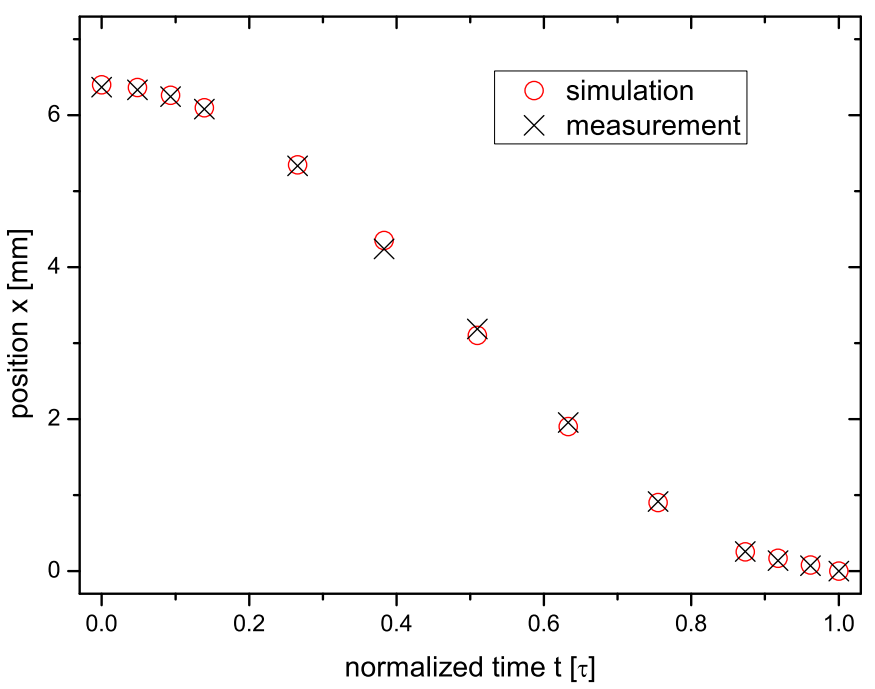

Fig. 4. Simulated position of the harmonic trap and measured positions of a sample of cold rubidium.

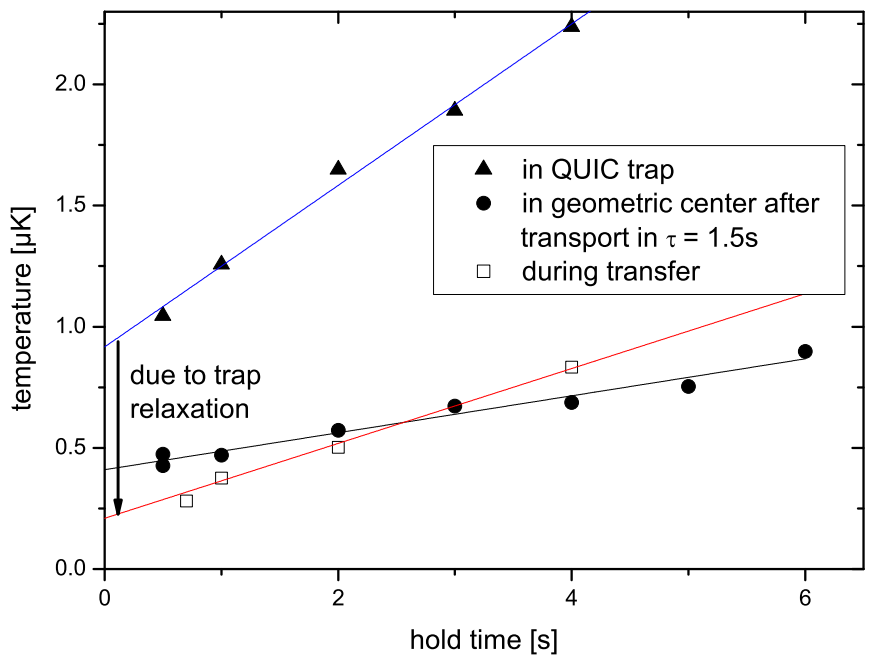

Fig. 5. Heating of a ${ }^{87} \mathrm{Rb}$ ensemble just above $T_{\mathrm{C}}$ in either a magnetic trap or while being transported. For the later, the temperature in the magnetic trap at the end of the transport was measured for different transport times $\tau$.

of the trap precisely, as illustrated in Fig. 4 The measurement confirms the simulated transport of the ultracold mixture. It shows that we have complete control over the position of the atoms by applying designed currents through the coils to induce an adiabatically changing magnetic field.

Due to the lower trapping frequencies the heating rate of the sample in the final trap is lower than in the initial QUIC trap (75 and $330 \mathrm{nK} / \mathrm{s})$. The observed heating during the transfer is between these two rates and thus no additional heating due to the transport is present as shown in Fig. 15. In fact, it is possible to transport quantum degenerate gases without loss of the degeneracy if the transport time $\tau$ is shorter than $1 \mathrm{~s}$.

Due to the chosen functional behavior [see Eq. (11)] in particular short transport times $\tau<1.5 \mathrm{~s}$ lead to an oscil-

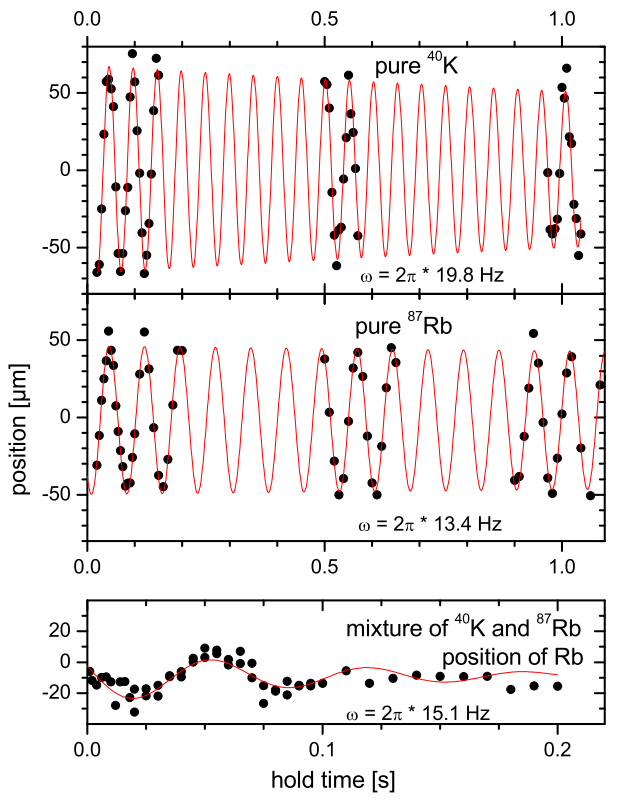

Fig. 6. Oscillations in the harmonic trap after the transfer from the QUIC trap to the geometric center for pure samples and a mixture of rubidium and potassium. Note the change in the time scale for the mixture, in which the oscillation of rubidium is not only smaller but also strongly damped by the potassium atoms.

lation of the atomic clouds in the final magnetic trap (see Fig. 61). In this case the trap is not changing adiabatically anymore and the atoms act like a classical particle in a harmonic trap. We have experimentally determined that twelve ramps represent a good compromise between adiabaticity of the transport and experimental complexity.

We obtain similar results for the transport of a heteronuclear mixture. Due to the different mass, the ${ }^{40} \mathrm{~K}$ atoms oscillate with a different frequency. We observed that the amplitude of the oscillations of a cold cloud of a single species is significantly reduced if $\mathrm{Rb}$ and $\mathrm{K}$ atoms are transported together (see Fig. 6). Similar effects in the hydrodynamic regime were observed and studied in greater detail in Ref. [4]. For transport times above $1.5 \mathrm{~s}$, no significant oscillations can be observed and the twelve ramps of the currents taken directly from the simulations need no fine adjustments.

In addition to the transfer mechanism our method allows us to accelerate and launch ultracold ensembles by quickly switching off all currents in the middle of the transfer. Figure 7 shows BECs launched with horizontal velocities of up to $80 \mathrm{~mm} / \mathrm{s}$. This technique is an alternative for launching cold ensembles with optical lattices [19] or detuned laser fields, which is usually applied in fountain clocks 24] or in inertial sensors 25145]. Also, the observation time of quantum degenerate gases can be doubled if the sample is launched against gravity, provided that the time of flight is the limiting factor. 


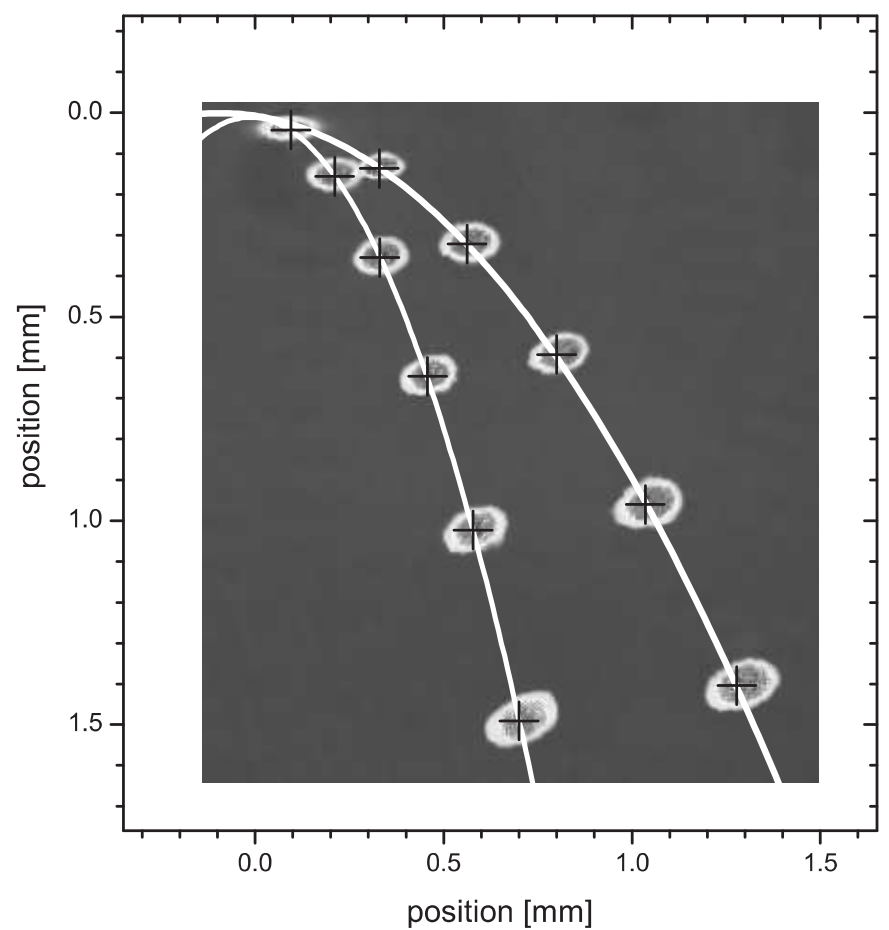

Fig. 7. Trajectories of a BEC launched with two different horizontal speeds. Several absorption images are compiled into a single image and overlay with two parabolas fitted to the obtained positions of the clouds. From these fits, velocities of $40 \mathrm{~mm} / \mathrm{s}$ and $80 \mathrm{~mm} / \mathrm{s}$ can be interfered, which agree with the calculated speed of the harmonic trap at the time when all currents are rapidly switched off.

\section{Summary and Outlook}

We have developed a transport mechanism for quantum degenerate gases in a harmonic trapping potential and we have demonstrated the simultaneous transport of quantum degenerate bosonic and fermionic samples over a distance of up to $6 \mathrm{~mm}$. This mechanism may be cascaded to cover even larger distances and thus enables magnetic transport experiments with large quantum degenerate samples in macroscopic trap configurations. This concept adds another powerful method to the toolbox of quantum atom optics and will allow novel designs for interferometric sensors and clocks.

This transport mechanism enriches the possible applications of the popular QUIC trap geometry significantly. It allows for a transport of a quantum degenerate sample to the geometric center of the main coil pair of the QUIC trap. This enables their use for the production of large homogeneous fields and thus the investigation and utilization of Feshbach resonances, e.g., for the creation of heteronuclear dimers or the tuning of the interaction of the two trapped isotopes. Moreover, the transport facilitates the optical access for additional beams, e.g., for the creation of optical lattices or optical pumping such as photoassociation.

In the case of our experiment, the modification has reduced the spread of a magnetic field of $500 \mathrm{G}$ from $240 \mathrm{mG}$ to below $16 \mathrm{mG}$ for ensembles at $1 \mu \mathrm{K}$. This variation of the magnetic field is no longer due to the inhomogeneity of the field but due to residual current noise. Such well controlled magnetic fields in combination with a dipole trap allow for the precise control of the effective interaction strength and open the pathway to studies of many particle physics such as the phase separation between Fermions and Bosons or molecular physics such as cold molecule production.

\section{Acknowledgments}

We acknowledge support from the Deutsche Forschungsgemeinschaft (SFB 407 and Graduate College Quantum Interference and Applications).

\section{References}

1. M. H. Anderson et al., Science 269, 198 (1995).

2. K. B. Davis et al., Phys. Rev. Lett. 74, 5202 (1995).

3. B. DeMarco, H. Rohner, and D. Jin, Rev. Scient. Instr. 70, 1967 (1999).

4. S. L. Rolston and W. D. Phillips, Nature 416, 219 (2002).

5. A. E. Leanhardt et al., Phys. Rev. Lett. 89, 040401 (2002).

6. D. Cassettari et al., Phys. Rev. Lett. 85, 5483 (2000).

7. D. Müller et al., Phys. Rev. A 63, 041602 (2001).

8. W. Hänsel, J. Reichel, P. Hommelhoff, and T. W. Hänsch, Phys. Rev. A 64, 063607 (2001).

9. T. Schumm et al., Nature Physics 1, 57 (2005).

10. Y.-J. Wang et al., Phys. Rev. Lett. 94, 090405 (2005).

11. M. Fattori et al., arxiv.org/abs/0710.5131 (2007).

12. G.-B. Jo et al., arxiv.org/abs/0706.4041 (2007).

13. G.-B. Jo et al., Phys. Rev. Lett. 98, 180401 (2007).

14. J. Billy et al., arxiv.org/abs/0712.1482 (2007).

15. E. Andersson et al., Phys. Rev. Lett. 88, 100401 (2002).

16. C. P. Search and P. Meystre, Phys. Rev. A 67, 061601 (2003).

17. G. Modugno et al., Fortschr. Phys. 52, 1173 (2004).

18. T. L. Gustavson et al., Phys. Rev. Lett. 88, 020401 (2001).

19. S. Schmid et al., New J. Phys. 8, 159 (2006).

20. W. Hänsel, P. Hommelhoff, T. W. Hänsch, and J. Reichel, Nature 413, 498 (2001).

21. P. Hommelhoff et al., New J. Phys. 7, 3 (2005).

22. J. Fortágh and C. Zimmermann, Rev. Mod. Phys. 79, 235 (2007).

23. T. Esslinger, I. Bloch, and T. W. Hänsch, Phys. Rev. A 58, R2664 (1998).

24. R. Wynands and S. Weyers, Metrologia 42, S64 (2005).

25. F. Yver-Leduc et al., J. Opt. B 5, S136 (2003).

26. R. Dumke et al., Phys. Rev. Lett. 89, 220402 (2002).

27. A. Günther et al., Phys. Rev. A 71, 063619 (2005).

28. S. Inouye et al., Nature 392, 151 (1998).

29. S. Inouye et al., Phys. Rev. Lett. 93, 183201 (2004).

30. F. Ferlaino et al., Phys. Rev. A 73, 40702 (2006).

31. C. Klempt et al., Phys. Rev. A 76, 020701 (2007).

32. C. Klempt et al., Phys. Rev. A 73, 13410 (2006).

33. B. DeMarco and D. S. Jin, Science 285, 1703 (1999).

34. J. N. Walpole, Opt. Quantum Electron. 28, 623 (1996).

35. B. P. Anderson and M. A. Kasevich, Phys. Rev. A 63, 023404 (2001). 
36. S. N. Atutov et al., Phys. Rev. A 67, 053401 (2003).

37. H. J. Lewandowski, D. M. Harber, D. L. Whitaker, and E. A. Cornell, J. Low Temp. Phys. 132, 309 (2003).

38. M. Greiner, I. Bloch, T. W. Hänsch, and T. Esslinger, Phys. Rev. A 63, 031401 (2001).

39. J. Goldwin et al., Phys. Rev. A 70, 21601 (2004).

40. J. F. Bertelsen, H. K. Andersen, S. Mai, and M. Budde, Phys. Rev. A 75, 013404 (2007).

41. M. Theis et al., Phys. Rev. Lett. 93, 123001 (2004).

42. T. Lahaye et al., Phys. Rev. A 74, 033622(R) (2006).

43. J. Weiner, V. S. Bagnato, S. Zilio, and P. S. Julienne, Rev. Mod. Phys. 71, 1 (1999).

44. F. Ferlaino et al., J. Opt. B 5, S3 (2003).

45. T. Müller et al., arxiv.org/abs/0705.4544 (2007). 